\title{
La disponibilidad léxica como instrumento de evaluación léxica de aprendices griegos de ELE
}

Palabras clave: disponibilidad léxica, aprendices griegos de ELE, competencia léxica, evaluación de la competencia léxica

\section{Introducción}

La prueba de la disponibilidad léxica se concibe como una herramienta de recogida de datos que pueden servir para distintos objetivos. Este trabajo pretende profundizar en la aplicación de esta técnica a la enseñanza del español como lengua extranjera (ELE) y específicamente a la evaluación de la competencia léxica de los estudiantes. En concreto, nuestro objetivo es comprobar si la prueba de disponibilidad léxica puede servir, por un lado, como herramienta de evaluación del desarrollo del tamaño del léxico productivo del área temática analizada, y por otro, como prueba de diagnóstico del conocimiento productivo del léxico temático analizado desde una perspectiva cuantitativa y cualitativa.

Antes de formular nuestras preguntas de investigación y presentar la metodología y los resultados de nuestro estudio, hacemos referencia a las principales cuestiones relacionadas con la evaluación de la competencia léxica, tras lo cual explicamos en qué consiste la prueba de la disponibilidad léxica y cómo se podría utilizar en la evaluación léxica. Estas teorías nos permiten alcanzar mayor profundidad en la comprensión del tema investigado, lo que nos ayudará a explicar e interpretar nuestros resultados.

\section{Evaluación de la competencia léxica}

A pesar de que el componente léxico está en el epicentro de la adquisición de una LE, los expertos expresan su preocupación por la desatención que ha 
sufrido el ámbito de la evaluación del léxico (Meara, 1980; Read, 1997 y 2000). Según López-Mezquita Molina (2005: 673), una de las razones a las que se debe este descuido es el enfoque comunicativo. Como afirma Schmitt (2000: 14), si bien en los métodos comunicativos se pone énfasis en que los alumnos desarrollen su competencia comunicativa, en la cual se incluye la competencia léxica, lo cierto es que en la práctica el léxico queda relegado a un segundo plano. Esto sucede, como explica López-Mezquita Molina (2005: 673), porque la lengua se concibe como un conjunto de destrezas y no se da énfasis a los elementos estructurales, como son la gramática y el léxico.

Sin embargo, como afirman varios investigadores (López-Mezquita Molina, 2005: 673; Hidalgo Gallardo, 2017: 48), los últimos años se ha revalorizado el papel del léxico en el desarrollo comunicativo, por lo que se ha prestado más atención también a su evaluación. Dicho cambio ha dado lugar a un gran número de publicaciones que se enfocan hacia diferentes cuestiones relacionadas con la evaluación léxica, por ejemplo, la de qué aspecto de la competencia léxica evaluar (Nation, 1990; 2001; Meara, 1996; Henriksen, 1999) y la de cómo evaluarlo (Read, 2000). Asimismo, el Marco común europeo de referencia (MCER) (Consejo de Europa, 2002) ofrece criterios de evaluación léxica proponiendo descriptores de cada nivel para la gradación de la riqueza y del dominio del vocabulario, presentados de manera aislada (las escalas ilustrativas de riqueza y dominio de vocabulario) y de manera integrada (los descriptores holísticos de niveles).

\section{1 ¿Qué evaluar?}

Una de las cuestiones principales que hay que tener en cuenta al evaluar la competencia léxica es que se trata de una competencia multidimensional, es decir, que tiene muchos componentes, por lo que antes de la evaluación es necesario determinar el constructo de la competencia léxica que se pretende evaluar. Como señala López-Mezquita Molina (2005: 679), para poder precisar el aspecto que se pretende evaluar hay que basarse en la descripción de la competencia léxica.

Muchos son los investigadores que han tratado de describir la competencia léxica. Todos ellos sostienen que para proceder a la descripción de dicha competencia es menester centrarse en las diferentes dimensiones de las unidades léxicas, dado que la competencia léxica consiste exactamente en el conocimiento de estas últimas y en su adecuado uso.

Las diferentes propuestas sugeridas por los investigadores han tenido como resultado la aparición de modelos diferentes que describen la competencia léxica. 
Aunque Meara (1996: 45) menciona que todo este trabajo realizado en relación con la descripción de la competencia léxica tiene como punto de partida el trabajo de Richards (1976), encontramos esfuerzos de discusión del concepto también en Fries (1945), quien, aunque en ningún momento hace uso del término competencia léxica, se refiere a los cuatro niveles de significado de las palabras: el contenido léxico de las mismas (o sea, la definición que encontramos en un diccionario), el significado sintáctico, el significado morfológico y la entonación que usa la persona que habla.

No obstante, es Richards (1976) quien propone un modelo descriptivo de la competencia léxica más sistemático, y por tal motivo, según Jiménez Catalán (2002: 153), su artículo ha generado muchos trabajos posteriores (Wallace, 1982; Carter, 1987; Nation, 1990, 2001; Cassany, Luna y Sanz, 1994; Higueras, 1996; Schmitt, 2000). Su modelo se basa en la descripción de los diferentes rasgos y aspectos de la unidad léxica. Según Richards (1976), conocer una palabra significa conocer la frecuencia de aparición de una palabra tanto en la lengua oral como en la escrita, conocer el uso adecuado de una palabra según la función y la situación, conocer el comportamiento sintáctico de la palabra, además de conocer la forma subyacente y las posibles derivaciones, las asociaciones, su valor semántico y sus diferentes significados. Asimismo Richards (1976) se refiere al desarrollo continuo del vocabulario a lo largo de toda la vida, su aprendizaje incesante y acumulativo.

El más importante de los trabajos que han seguido esta línea de investigación, es decir, la que se centra en la enumeración de los componentes de la competencia léxica, es el llevado a cabo por Nation $(1990,2001)$. Nation $(1990,2001)$ hace una distinción entre el nivel receptivo y el nivel productivo que presuponen distintos tipos de conocimiento, tomando en cuenta que en cada nivel se realizan distintos procesos cognitivos y se activan procesos de aprendizaje diferentes, como apunta Jiménez Catalán (2002: 158). Los componentes del conocimiento receptivo, según Nation (1990, 2001), son el reconocimiento de las formas escrita y oral, el conocimiento de las relaciones gramaticales con otras palabras, así como las colocaciones, la frecuencia de aparición de la palabra y su uso adecuado según el contexto (registro, estilo) y, por último, el conocimiento del concepto que representa y las asociaciones con otras palabras. El conocimiento productivo, por otro lado, presupone la facultad de pronunciar y escribir adecuadamente una palabra, de usarla de manera gramaticalmente correcta, de utilizar bien las colocaciones, de emplearla en los contextos adecuados y de sustituirla apropiadamente si es posible.

Por otro lado, algunos investigadores critican la descripción de la competencia léxica basada en la mera enumeración de las dimensiones de las unidades 
léxicas y proponen frente a esto otras alternativas. Uno de ellos es Meara (1996: 35-53), quien piensa que la utilización de este tipo de modelos para describir la competencia léxica puede causar problemas prácticos a los investigadores, dado que es casi imposible evaluar todos estos componentes con el fin de elaborar estimaciones de la competencia léxica.

Asimismo, Meara sostiene que todos los modelos que se parecen al de Richards (1976) se centran en aspectos de palabras aisladas, y no pueden generalizarse ni aplicarse a la totalidad del léxico de una persona. Sin embargo, hay que decir que el investigador que desea evaluar la competencia léxica de un sujeto no suele fijarse en palabras aisladas, sino en su léxico entero.

En consecuencia, Meara (1996: 35-53) plantea una alternativa, proponiendo dos dimensiones de la competencia léxica que considera globales: el tamaño del vocabulario y su organización. Desde su perspectiva, estas dos dimensiones caracterizan la competencia léxica como una entidad y facilitan la evaluación de la misma. Según su opinión, es más práctico y eficaz considerar la competencia léxica como un conjunto de características globales que como una acumulación de características de palabras aisladas.

Otro investigador reacio a los modelos que presentan la competencia léxica como una lista de dimensiones de las palabras es Henriksen (1999). En su intento de ser más específico sugiere tres dimensiones de la competencia léxica: el conocimiento de lo parcial y de lo preciso, la riqueza del conocimiento, en el sentido de la organización del léxico de Meara (1996: 47), y el conocimiento de lo receptivo y de lo productivo.

Vemos, para concluir, que no hay unanimidad entre los estudiosos en cuanto a la descripción de la competencia léxica. Sin embargo, el estudio de estos modelos nos ayuda a entender la complejidad de la competencia léxica y la necesidad de precisar el aspecto que queremos evaluar antes de proceder a cualquier tipo de evaluación de dicha competencia.

\section{2 ¿Cómo evaluar?}

Tras la selección del aspecto de la competencia léxica y el tipo de vocabulario que queremos evaluar, tenemos que especificar el propósito de la evaluación y decidir sobre el modo adecuado para llevar a cabo la misma. Dichas decisiones son cruciales porque van a determinar el tipo de prueba y el tipo de ítem que debemos emplear. 
Con respecto al propósito al que podría servir una evaluación, las categorías de las pruebas mencionadas por López-Mezquita Molina (2005: 538) son las siguientes: las pruebas de dominio (que evalúan la competencia de los estudiantes en diferentes campos del aprendizaje de LE para comprobar si han adquirido un cierto nivel de competencia), las pruebas de aprovechamiento (cuyo objetivo es confirmar si los alumnos han alcanzado los objetivos previstos), las pruebas de diagnóstico (que pretenden identificar las lagunas de los aprendices) y finalmente las pruebas de nivel (las cuales evalúan el nivel lingüístico de los alumnos con el fin de asignarlos a la clase apropiada).

En cuanto a la manera de evaluar el léxico, según la clasificación propuesta por Read (2000: 9), la primera decisión que hay que tomar es si el conocimiento léxico se va a evaluar independientemente o si se va a medir dentro de otras destrezas. Cuando evaluamos el léxico como un constructo independiente usamos ítems independientes, mientras que cuando medimos el léxico dentro de otro constructo utilizamos ítems integrados, y por su parte el vocabulario contribuye a la evaluación de un constructo mayor, por ejemplo, la comprensión de lectura o la producción escrita.

La segunda decisión que tenemos que tomar es si se va a evaluar una lista concreta de palabras, seleccionadas según algún criterio (p.e. la frecuencia), o si se va a evaluar el vocabulario incluido en un determinado texto. En el primer caso, contamos con ítems selectivos frente a los globales, preferibles cuando se pretende medir todo el léxico contenido en un texto.

La tercera y última cuestión que hay que aclarar al evaluar el léxico es decidir si se va a realizar fuera o dentro de contexto. En el primer caso, usamos ítems descontextualizados, mientras que en el segundo los ítems aparecen contextualizados, así que los candidatos tienen que basarse en el contexto para contestar.

La evaluación del léxico como un constructo independiente y fuera de contexto y el uso de ítems independientes y descontextualizados facilitan la evaluación de las dimensiones de la competencia léxica separadamente. Así pues, basándonos en la propuesta de Meara (1996: 47), hay ítems que miden la extensión del vocabulario (de selección múltiple, las listas sí/no, verdadero/falso) e ítems que miden su profundidad (asociaciones, escalas).

Para concluir, se puede observar que hay muchas maneras diferentes de medir las distintas dimensiones de la competencia léxica. El estudio de todos estos tipos de pruebas e ítems nos puede ayudar a decidir cuál es el modo adecuado a la hora de medir el aspecto de la competencia léxica que nos interesa. 


\subsection{La disponibilidad léxica como método de evaluación de la competencia léxica de estudiantes de LE}

Según Bartol Hernández (2006: 379), la prueba de la disponibilidad léxica es una prueba asociativa que estimula el lexicón mental de los informantes con vistas a que produzcan las palabras más disponibles que les vienen a la mente cuando se les presenta la palabra estímulo. De esta manera, como afirma López Morales (1984: 62), el léxico disponible se compone de las primeras unidades léxicas producidas ante un estímulo temático concreto.

Según Tomé Cornejo (2015: 344-345), y basándonos en la propuesta de Meara (1996: 47), la prueba de la disponibilidad léxica nos permite evaluar tanto la extensión como la profundidad del conocimiento léxico. En concreto, puede ofrecer una estimación del tamaño del léxico temático conocido y además nos permite evaluar algunos aspectos de la profundidad del conocimiento léxico, como el conocimiento de la forma de la palabra (ortografía o pronunciación ${ }^{15}$ de la misma) y la vinculación de esta con el campo semántico analizado. Además, un análisis minucioso de las respuestas de los estudiantes nos puede aportar información sobre el uso de las estrategias de evocación que realizan (clusters y switches), lo cual nos permite ver la organización de estas palabras en su lexicón mental. De hecho, son varios los trabajos que se inscriben en esta línea, entre ellos los de Ferreira y Echeverría (2010: 133-153), Sánchez-Saus Laserna, (2011: 323-399), Tomé Cornejo (2015: 80-104) y Palapanidi (2019: 1-10).

Además, como sostienen varios investigadores, entre ellos Paredes García (2015: 22) y Tomé Cornejo (2015:341-342), la aplicación de este tipo de prueba a aprendices de LE puede ser explotada para la evaluación de su competencia léxica con distintos propósitos.

Por una parte, esta prueba nos permite observar la evolución de la competencia léxica de estudiantes de LE ya que ofrece información sobre el tamaño del léxico temático más inmediato que ellos poseen, por lo cual se hace posible la comparación en distintas fases del aprendizaje. Varios son los que han seguido esta línea de investigación. Algunos de ellos son Carcedo González (1998: 205224), López González (2010: 1-13), Šifrar Kalan (2014: 63-84) y Palapanidi (2016: 305-313).

Además, la comparación de las respuestas de los estudiantes de LE con las de nativos o con el Plan Curricular del Instituto Cervantes (PCIC) nos permite identificar lagunas en la competencia léxica de los aprendices de LE. Algunos

15 Solo en el caso de recoger los datos de manera oral. 
de los estudios que comparan el léxico disponible de estudiantes de ELE con el propio de nativos son el estudio de Šifrar Kalan (2012: 1-19), el de Caggiula (2013: 167-229) y el de Palapanidi y Agustín Llach (2017: 297-306). Mientras que entre los estudios que comparan el léxico disponible de estudiantes de ELE con el PCIC destacan el de Zuccalà (2014: 1-16), el de Paredes García (2015: 1-32) y el de Hidalgo Gallardo (2017: 43-63).

Teniendo en cuenta las dimensiones de la competencia léxica, las clasificaciones de las pruebas expuestas y los estudios mencionados, la prueba de la disponibilidad léxica podría considerarse un instrumento de evaluación del desarrollo del tamaño del léxico productivo y también una herramienta de diagnóstico del vocabulario productivo de naturaleza independiente, descontextualizada discursivamente. Con el fin de verificar la primera de nuestras hipótesis, comparamos el léxico disponible de dos grupos de aprendices griegos de ELE de diferente nivel lingüístico ( $\mathrm{B}_{1}$ y $\mathrm{C}_{1}$ ), y para comprobar nuestra segunda hipótesis cotejamos las respuestas de nuestros informantes de los dos niveles diferentes (B1 y $\mathrm{C}_{1}$ ) con las "Nociones específicas" del Plan Curricular del Instituto Cervantes (PCIC) de los niveles $\mathrm{A}_{1}-\mathrm{A}_{2}$ y $\mathrm{B}_{1}-\mathrm{B}_{2}$ respectivamente. De esta manera, se pueden formular las siguientes preguntas de investigación:

- ¿Puede la prueba de disponibilidad léxica servir como herramienta de evaluación del desarrollo del tamaño del léxico productivo en el centro de interés "La casa" de aprendices griegos de ELE?

- ¿Puede la prueba de disponibilidad léxica servir como prueba de diagnóstico del léxico productivo en el centro de interés "La casa" de aprendices griegos de ELE desde una perspectiva cuantitativa y una perspectiva cualitativa?

\section{Metodología}

\subsection{Muestra}

Se recogieron datos de 71 estudiantes de Filología Hispánica de la Universidad Nacional y Kapodistríaca de Atenas. Los estudiantes fueron clasificados tras los resultados de un examen de nivel. En el nivel B1 se incluyeron los estudiantes que sacaron una puntuación de entre 30 y 40, y en el nivel C1 a los que obtuvieron entre 50 y 60 . De tal manera, tras la corrección de las pruebas, se clasificaron 35 participantes en el nivel B1 y 36 participantes en el nivel C1. 
Se trata de una muestra bastante homogénea, dado que todos los participantes son adultos (18-25 años), poseen la misma lengua materna, el griego, y prevalece el sexo femenino. En cuanto a su perfil lingüístico, todos conocen otras LE, aparte de su LM y el español. Todos los informantes del grupo del nivel B1 conocen una LE (inglés), 17 informantes hablan dos LE (inglés y francés o alemán o italiano) y 4 hablan tres LE (inglés, francés e italiano o alemán). Todos los del nivel C1 tienen conocimientos de una LE (inglés), 18 hablan dos LE (inglés y francés o alemán o italiano) y 7 hablan tres LE (inglés, francés e italiano o alemán). Dicha información se presenta en la Tabla 1.

\begin{tabular}{|l|l|c|c|c|}
\hline \multirow{2}{*}{ Nivel lingüístico } & \multirow{2}{*}{ Número de participantes } & \multicolumn{3}{|c|}{ LE conocidas } \\
\cline { 3 - 5 } & & 1LE & 2LE & 3LE \\
\hline B1 & 35 & 35 & 17 & 4 \\
\hline C1 & 36 & 36 & 18 & 7 \\
\hline
\end{tabular}

Tabla 1. El perfil lingüístico de los participantes

\subsection{Materiales}

\subsubsection{Prueba de disponibilidad léxica}

Los datos recogidos son de varios centros de interés. No obstante, nos limitaremos a analizar uno: "La casa". Se ha seleccionado dicho centro de interés puesto que es un área temática que aparece en todos los manuales de ELE de los niveles iniciales $\left(\mathrm{A}_{1}-\mathrm{A}_{2}\right)$ y de los intermedios $\left(\mathrm{B}_{1}-\mathrm{B}_{2}\right)$, sirviendo por ello al objetivo de este estudio.

\subsubsection{Examen de nivel}

El examen de nivel que se utilizó para la clasificación de los participantes contenía 80 preguntas de selección múltiple. Es uno de los modelos de exámenes de Gozalo y Martín (2008: 49-55) que están diseñados para determinar el nivel lingüístico de estudiantes de ELE.

\subsubsection{Dispolex}

Para informatizar los datos hemos utilizado el programa DISPOLEX. Se trata de un programa informático que ofrece acceso gratuito a las herramientas necesarias para hacer los cálculos de la disponibilidad léxica. 


\subsubsection{Plan Curricular del Instituto Cervantes (PCIC)}

Con el fin de detectar las carencias léxicas en el área temática analizada, se han comparado las respuestas de los participantes con el capítulo 9 ("Nociones específicas" Inventario A1-A2 e Inventario B1-B2) del PCIC.

\subsection{Primera fase del estudio: Prueba de disponibilidad léxica}

\subsubsection{Procedimiento}

Siguiendo las pautas metodológicas de otros estudios parecidos, hemos dado a los informantes dos minutos de tiempo para cada área temática, a fin de que produjeran por escrito todas las palabras relacionadas con el área en cuestión que les venían a la mente.

\subsubsection{Edición y procesamiento de los datos}

Los datos fueron recogidos el mes de octubre de 2017 durante la hora de clase. En lo concerniente a la edición de nuestros datos, hemos seguido las normas comúnmente usadas en estudios de este tipo, indicadas en Samper Padilla (1998). Esto es, se han eliminado las respuestas repetidas y se han aceptado las palabras con algún error ortográfico, las cuales hemos corregido siguiendo las reglas ortográficas del español. Finalmente, se han neutralizado las variantes flexivas, es decir, se ha introducido en el programa informático el infinitivo de los verbos, así como el masculino de los adjetivos.

Tras la edición de nuestros datos, hemos procedido a transmitirlos al programa informático DISPOLEX. Hemos pasado todas las respuestas de cada participante por grupo y por centro de interés, teniendo en cuenta la posición de cada palabra dentro de todas las respuestas, es decir, el orden de producción de cada una de ellas. De esta manera hemos podido hacer los cálculos necesarios.

\subsection{Segunda fase del estudio: Comparación con las "Nociones específicas" del PCIC}

Para detectar las carencias de nuestros participantes de nivel $\mathrm{B}_{1}$ en el área temática "La casa" hemos comparado sus respuestas con las palabras del área temática número 10, denominada "Vivienda" e incluida en "Nociones específicas" Inventario $\mathrm{A}_{1}-\mathrm{A}_{2}$, mientras que para los informantes del nivel $\mathrm{C}_{1}$ hemos comparado sus respuestas con el Inventario $\mathrm{A}_{1}-\mathrm{A}_{2}$ y $\mathrm{B}_{1}-\mathrm{B}_{2}$ del PCIC. Para 
realizar dicha comparación, nos hemos basado en los subtemas propuestos por el PCIC. En efecto, dicha área temática se compone de algunos subtemas, que son los siguientes: Acciones relacionadas con la vivienda, Características de la vivienda, Actividades domésticas, Objetos domésticos. De acuerdo a estos subtemas, hemos clasificado las respuestas de nuestros estudiantes siguiendo el índice de disponibilidad. No hemos tomado en cuenta las respuestas que han sido producidas únicamente por un participante (porcentaje de aparición $<2,86 \%$ ), dado que la importancia de estas repuestas es escasa y estos ejemplos no pueden formar parte del conocimiento general de los dos grupos sobre este centro de interés.

\section{Resultados}

\subsection{La evolución del léxico disponible de los aprendices griegos de español como LE}

Con el fin de observar la evolución del conocimiento léxico de nuestros informantes en el área temática analizada, hemos calculado el número y la media de palabras y vocablos producidos. Los resultados de este análisis se pueden contemplar en la Tabla 2. Dichos resultados muestran que hay mayor productividad de palabras en el nivel más avanzado. Adicionalmente, esta mayor productividad de palabras se ve acompañada de una mayor variedad de respuestas, donde se observa una mayor media de vocablos producidos en los participantes que pertenecen al nivel lingüístico avanzado.

\begin{tabular}{|l|c|c|c|c|c|c|c|c|c|c|c|}
\hline \multirow{2}{*}{ Grupos } & \multicolumn{4}{|c|}{ Palabras } & \multicolumn{4}{c|}{ Vocablos } & I.C. \\
\cline { 2 - 14 } & Núm. & Med. & D.T. & Mín. & Max. & Núm. & Med. & D.T. & Mín. & Max. & \\
\hline Nivel B1 & 349 & 9,97 & 5,71 & 3 & 28 & 144 & 4,11 & 2,59 & 1 & 13 & 0,07 \\
\hline Nivel C1 & 491 & 13,64 & 5,61 & 4 & 28 & 206 & 5,72 & 2,8 & 1 & 12 & 0,07 \\
\hline
\end{tabular}

Tabla 2. El léxico disponible de los participantes en el centro de interés "La casa".

Para comprobar si los datos presentan una distribución normal, se aplicó la prueba de normalidad Shapiro-Wilk. Los resultados mostraron que no se cumple el supuesto de distribución normal para las palabras y vocablos de los alumnos del nivel B ( $p s<.001)$. Por tanto, para la comparación de medias se ha usado una prueba no paramétrica, la prueba U de Mann-Whitney. Los resultados revelaron diferencias estadísticamente significativas tanto en el número de vocablos $(Z=-2.624, p=.009)$ como en el número de palabras 
$(Z=-3.00, p=.003)$, siendo los alumnos del nivel $\mathrm{C}$ los que emplearon un mayor número de ambos.

\subsection{Comparación del léxico disponible de los aprendices griegos con las "Nociones específicas” del PCIC}

Con el fin de detectar las lagunas en el conocimiento léxico del área temática analizada, comparamos los vocablos más disponibles y producidos por más de un participante ( 50 en el nivel $\mathrm{B}_{1}$ y 55 en el nivel $\mathrm{C}_{1}$ ) con las entradas del PCIC de una manera cuantitativa y cualitativa.

\subsubsection{Análisis cuantitativo}

Para comparar el léxico disponible de los participantes y el PCIC, se calculan primero los vocablos exclusivos del PCIC por niveles. A continuación, se calculan los vocablos que se han producido exclusivamente en la prueba de la disponibilidad léxica en cada uno de los niveles ( $\mathrm{B}_{1}$ y $\mathrm{C}_{1}$ ), así como los vocablos comunes entre el PCIC y el nivel B1 y entre el PCIC y el nivel $\mathrm{C} 1$, y sus porcentajes en relación con el total de los vocablos más disponibles respectivamente. Los resultados presentados en la Tabla 3 muestran que las respuestas de los estudiantes del nivel más avanzado coinciden más con las palabras que aparecen en el PCIC.

\begin{tabular}{|l|l|c|c|c|c|c|c|c|c|l|}
\hline \multicolumn{3}{|l|}{$\begin{array}{l}\text { Vocablos exclusi- } \\
\text { vos PCIC }\end{array}$} & \multicolumn{3}{l|}{ Vocablos exclusivos D.L. } & \multicolumn{3}{l|}{ Vocablos comunes entre D.L.y } \\
PCIC
\end{tabular}

Tabla 3. Comparación cuantitativa entre el léxico más disponible en el centro de interés "La casa" y "Vivienda" de las "Nociones específicas" del PCIC.

\subsubsection{Análisis cualitativo}

A continuación, en las tablas 4-7, se presenta la comparación cualitativa de las respuestas más disponibles de nuestros informantes con las palabras del área temática "Vivienda" del PCIC. En los subtemas se ha incluido la categoría Otros, en la que hemos incluido respuestas de nuestros informantes que 
VERBA HISPANICA XXVII • NUEVOS RETOS Y PERSPECTIVAS EN LA ENSEÑANZA...

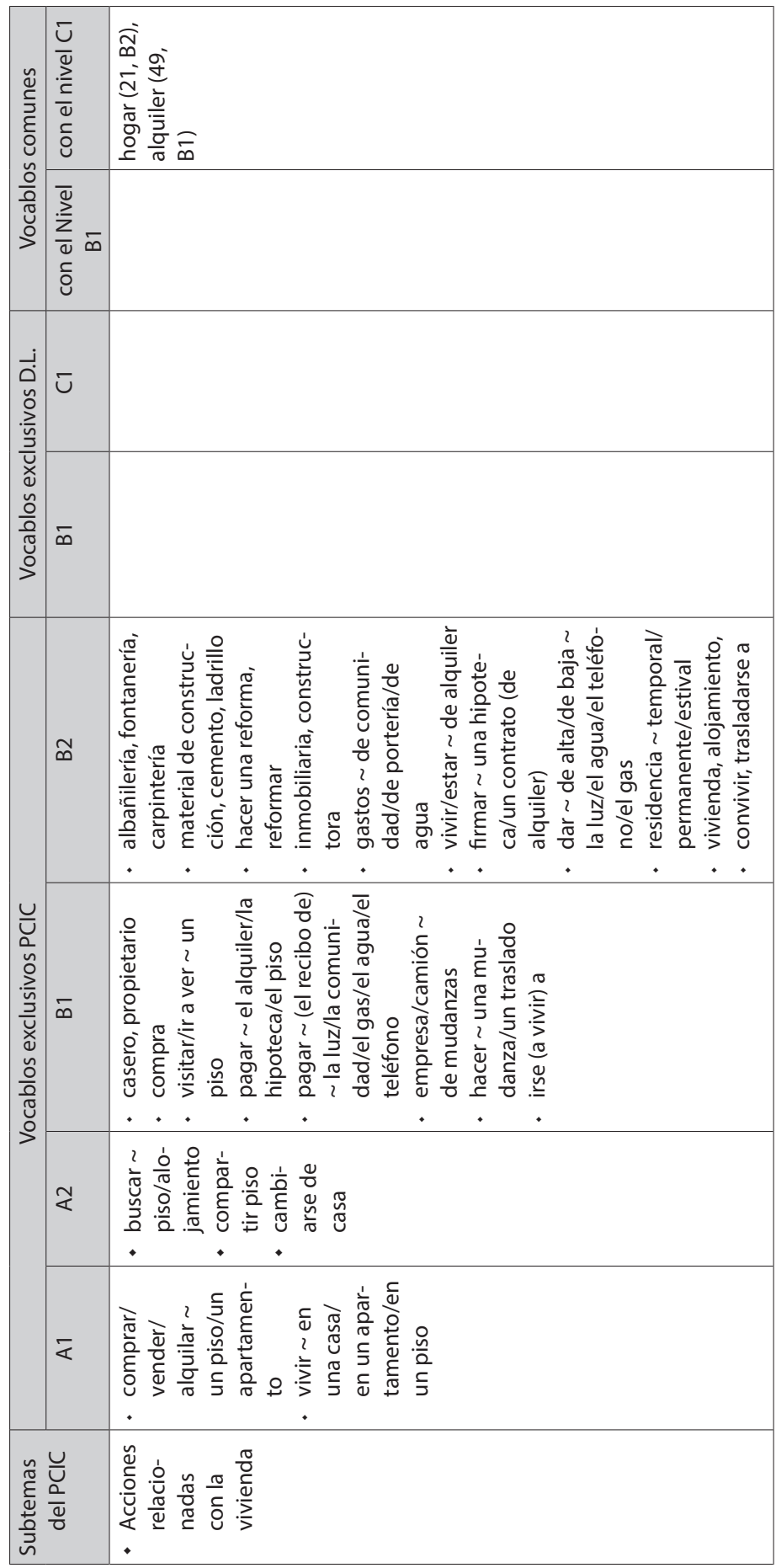

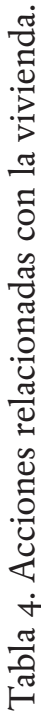




\begin{tabular}{|c|c|c|}
\hline \multirow{2}{*}{ 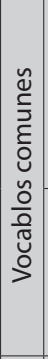 } & 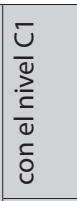 & 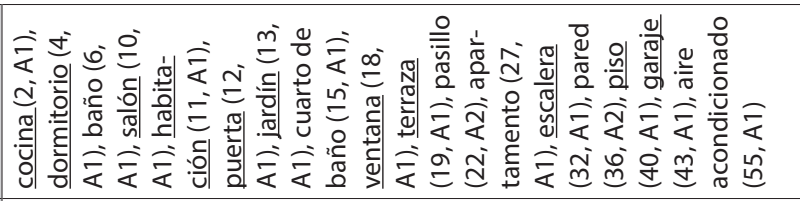 \\
\hline & $\begin{array}{l}\bar{\Sigma} \\
\frac{\bar{z}}{\alpha} \bar{\infty} \\
\bar{\delta}\end{array}$ & 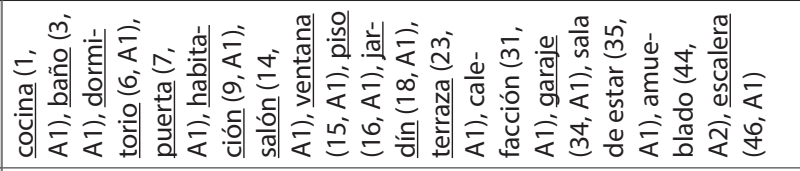 \\
\hline \multirow{2}{*}{ 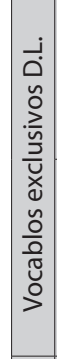 } & $\bar{v}$ & 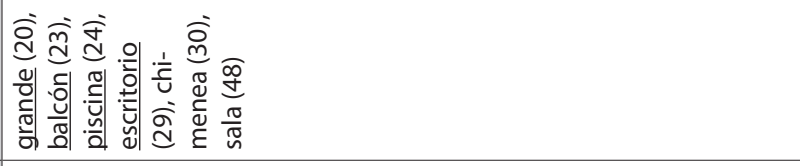 \\
\hline & $\bar{\infty}$ & 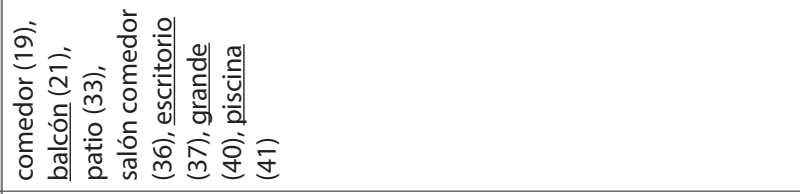 \\
\hline \multirow{4}{*}{ 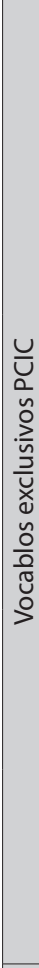 } & ఐี & 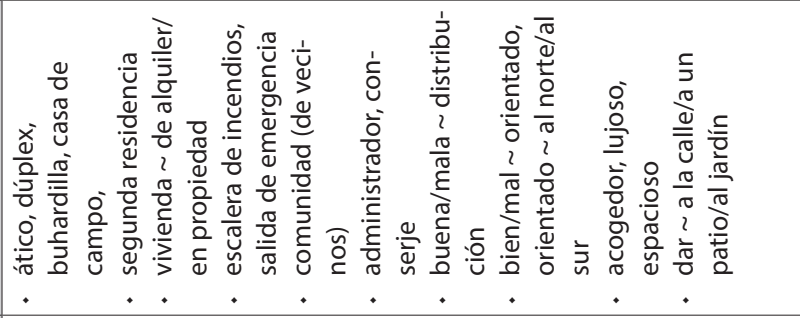 \\
\hline & $\bar{\infty}$ & 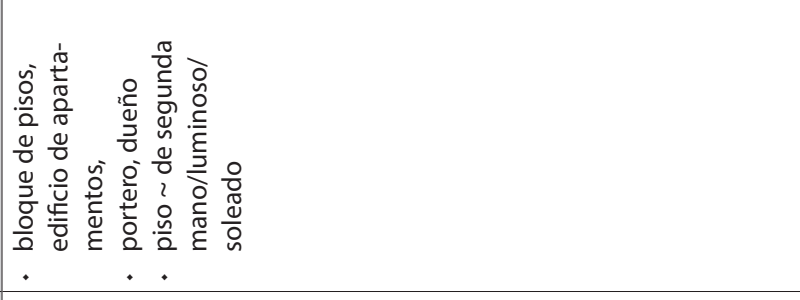 \\
\hline & $\stackrel{2}{\longleftarrow}$ & 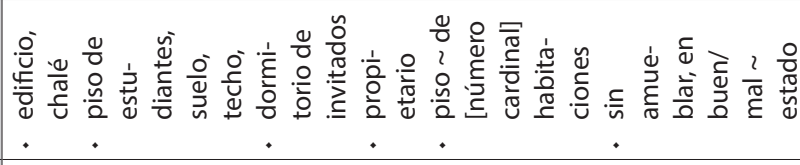 \\
\hline & $\bar{\alpha}$ & 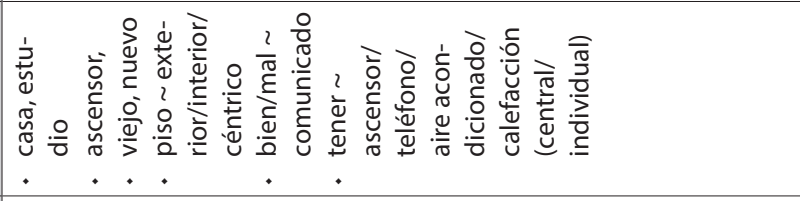 \\
\hline & & 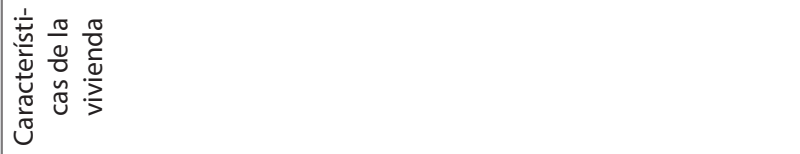 \\
\hline
\end{tabular}


VERBA HISPANICA XXVII • NUEVOS RETOS Y PERSPECTIVAS EN LA ENSEÑANZA...

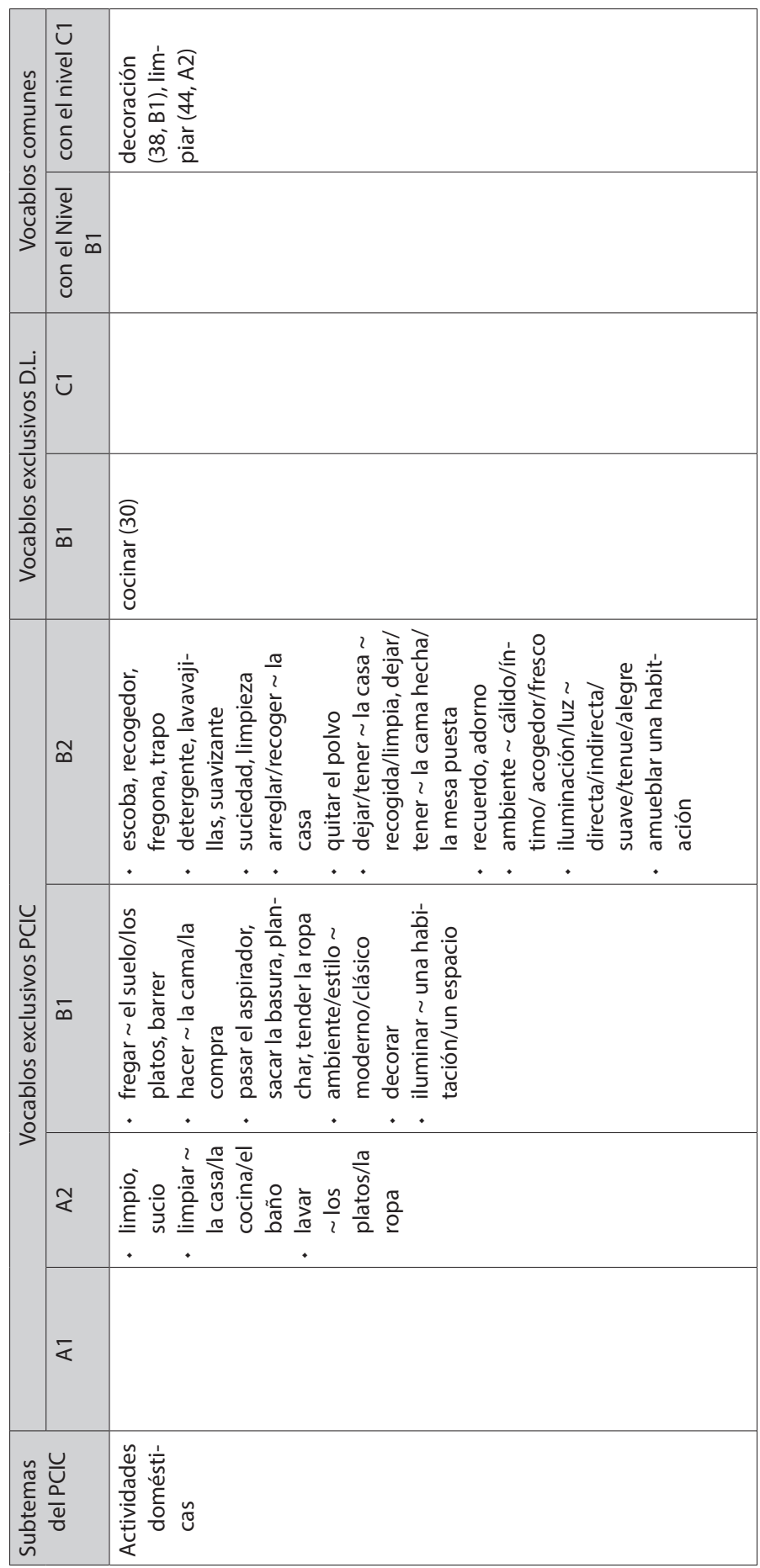

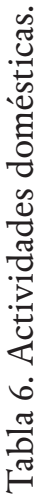




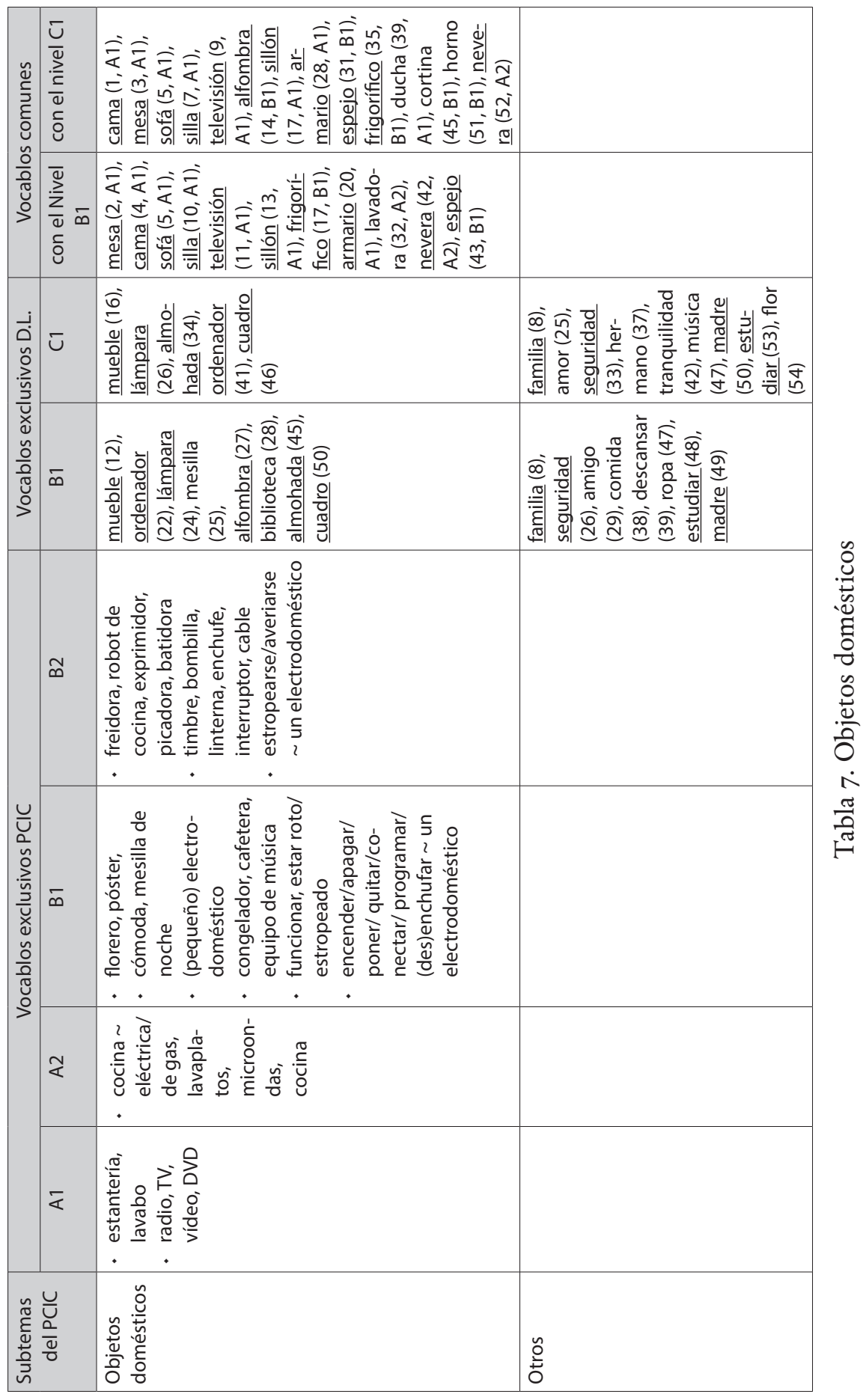


no pueden adherirse a ninguno de los subtemas propuestos por el PCIC. Se presentan los vocablos que aparecen exclusivamente en el PCIC por niveles, los vocablos producidos solo por nuestros informantes por niveles y también los vocablos comunes entre el PCIC y el nivel B1 y entre el PCIC y el nivel C1. El número entre paréntesis indica el orden de aparición de la palabra en la lista de la disponibilidad léxica y el nivel lingüístico en el que aparece en el PCIC. Las palabras comunes entre los dos grupos de participantes aparecen subrayadas, mientras que en negrita hemos señalado las palabras del PCIC que se repiten dentro del mismo tema.

\section{Discusión}

Los resultados de la primera parte del estudio revelan que a medida que avanza el nivel lingüístico de los informantes se observa tanto mayor producción de palabras como mayor diversidad léxica. El resultado obtenido coincide con los de estudios similares. En concreto, López González (2010) analiza el léxico disponible de alumnos polacos de secundaria que aprenden español como $\mathrm{LE}$ en dos niveles lingüísticos diferentes ( $\mathrm{B}_{1}$ y $\mathrm{B}_{2}+$ ). En la mayoría de los dieciséis centros de interés analizados, en los que se incluye "Las partes de la casa”, se observa un aumento en la producción de palabras y vocablos. La misma tendencia se observa también en el trabajo de Šifrar Kalan (2014), quien compara la disponibilidad léxica en español como LE de alumnos eslovenos de secundaria con un nivel aproximado a $\mathrm{B} 1$ con estudiantes universitarios con nivel $\mathrm{B}_{2}+$ en once centros de interés, incluido entre ellos "La casa". En todos los centros de interés se ha producido mayor cantidad de palabras y vocablos en el nivel más avanzado.

Dicha tendenia era de esperar, pues varios son los estudiosos (Ringbom, 1983; Meara, 2007) que afirman que conforme avanza el nivel lingüístico en LE, se desarrolla también la competencia léxica y se enriquece el lexicón mental. Por un lado, se van incorporando nuevas palabras en el lexicón mental y, por otro, se posibilitan más asociaciones tanto entre las nuevas unidades léxicas como entre las nuevas y las ya existentes. La información revelada es muy valiosa y nos permite afirmar que la prueba de la disponibilidad léxica puede servir como herramienta de evaluación del desarrollo del tamaño del léxico productivo del área temática analizada.

Pasando a los resultados de la comparación cuantitativa entre las respuestas más disponibles y producidas por más de un informante con las palabras del 
área temática "Vivienda" del PCIC, estos nos indican que el porcentaje de los vocablos producidos exclusivamente por los alumnos griegos es bastante elevado especialmente para el nivel $\mathrm{B}_{1}$ ( $48 \%$ ), mientras que es más bajo para el nivel $\mathrm{C}_{1}(36,3 \%)$. Este resultado podría justificarse si nos basamos en Ringbom (2007: 27-28), quien afirma que los aprendices tienden a producir respuestas raras y no estereotipadas, porque las asociaciones que realizan en su lexicón mental son diferentes a las de los nativos.

Sin embargo, el alto porcentaje de los vocablos exclusivos de la prueba de la disponibilidad léxica se puede explicar si los examinamos minuciosamente. Los resultados del análisis cualitativo muestran que algunos de estos son el resultado de asociaciones enciclopédicas, es decir, se deben a experiencias personales de los estudiantes (familia, seguridad, amigo, descansar, ropa, estudiar, madre, amor, bermano, tranquilidad, música, flor) y no son palabras del área temática "Vivienda", por lo que parece lógico que no estén incluidos en el PCIC.

Por su parte, sí se han producido algunas palabras relacionadas con los diferentes subtemas del PCIC, por ejemplo comedor, grande, balcón, patio, salón comedor, escritorio, piscina, chimenea, sala (Características de la vivienda), cocinar (Acciones domésticas) y mueble, ordenador, lámpara, almobada, cuadro, mesilla (Objetos domésticos). Pensamos que estas palabras son adecuadas para los estudiantes de ELE y por eso se deberían incluir en el PCIC.

Resultados similares se han encontrado también en otros estudios, como el de Hidalgo Gallardo (2017: 59), quien comparó las respuestas de sus informantes en el centro de interés "Aspecto físico" con "Partes del cuerpo" de las nociones específicas del PCIC. Su comparación reveló que sus informantes produjeron palabras que se ausentaban del PCIC pero que podrían encajar perfectamente en estas listas. Ausencias injustificables de unidades léxicas del PCIC localizó también Paredes García (2015: 15) tras la comparación del léxico disponible de informantes nativos con el PCIC. Adicionalmente, el mismo investigador comparando el léxico disponible de nativos, de estudiantes de español como LE y del PCIC, llegó a la conclusión de que en el PCIC aparecen unidades léxicas que no han sido producidas ni por los nativos ni por los estudiantes. Analizando los vocablos que se ausentan en la producción de los estudiantes griegos, observamos que la mayoría de estos pertenecen a dos subtemas (Acciones relacionadas con la vivienda y Actividades domésticas) y son verbos (buscar piso/ alojamiento, compartir piso, cambiarse de casa, etc.). En el primer subtema solo dos vocablos han sido producidos por los estudiantes del nivel $\mathrm{C} 1$, y en el segundo solo tres, uno por los del nivel B1 y dos por los del nivel C1. Además, notamos 
que muchos de los vocablos que faltan de la producción de los alumnos griegos son adjetivos (viejo, nuevo, limpio, sucio, luminoso, soleado, etc.).

Esta ausencia de verbos y adjetivos de nuestras listas de disponibilidad léxica y la prevalencia de los sustantivos coinciden con los resultados del estudio de Hidalgo Gallardo (2017), quien compara el léxico disponible de aprendices sinohablantes de español como LE en el centro de interés "El cuerpo humano" con las Nociones específicas del PCIC.

Pensamos que estos resultados no se deben exclusivamente a la falta de conocimientos por parte de los estudiantes griegos, sino también a la tendencia de los encuestados a omitir verbos y adjetivos y de producir sustantivos en las pruebas de disponibilidad léxica. El fenómeno de la omisión de verbos y adjetivos es señalado por Salazar García (2004: 258). De la misma manera, la tendencia a producir solo sustantivos es afirmada por muchos investigadores (Sánchez Gómez, 2005; Šifrar Kalan, 2014; Tomé Cornejo, 2015). Los dos fenómenos se deben principalmente a la configuración del centro de interés.

Por eso, si queremos localizar las carencias en el conocimiento léxico de un grupo de alumnos utilizando la prueba de disponibilidad léxica, tenemos que elegir muy cuidadosamente el centro de interés, de una manera que permita la producción de todas las categorías gramaticales de las palabras y no solamente los sustantivos. Hablando del centro de interés analizado en este estudio ("La casa"), pensamos que los resultados revelarían más información en cuanto a las lagunas en el conocimiento de los informantes si se hubieran utilizado como centros de interés los diferentes subtemas de "Vivienda" del PCIC.

En cuanto a los vocablos comunes con el PCIC, de particular interés es el hecho de que el $77,1 \%$ de estos pertenece al nivel $A_{1}$, aunque los informantes tienen un nivel bastante más alto ( $\mathrm{B}_{1}$ y $\mathrm{C}_{1}$ ). Solo aparecen seis vocablos de nivel $\mathrm{A}_{2}$, siete de $\mathrm{B}_{1}$ y uno de $\mathrm{B}_{2}$. Es un resultado encontrado también en otros estudios que comparan las listas de disponibilidad léxica con el PCIC, como el de Zuccalà (2014) y el de Hidalgo Gallardo (2017). Dado este hecho, a primera vista podríamos decir que la prueba de disponibilidad léxica es adecuada para localizar carencias léxicas solo en niveles iniciales. Sin embargo, pensamos que estos resultados se deben a la elección del centro de interés. La utilización de un centro de interés más específico permitiría la producción de vocablos menos básicos y generales, como también lo afirma Tomé Cornejo (2015:405).

Finalmente, observamos que en algunos casos se nota que se han activado vocablos aislados (limpiar), al tiempo que faltan vocablos que pertenecen al 
mismo campo semántico (fregar el suelo, barrer, pasar el aspirador, etc.). Este fenómeno nos muestra que las palabras activadas están almacenadas en el lexicón mental de los alumnos de forma aislada y que no se han formado redes semánticas, lo que, tal y como afirman Ferreira y Echeverría (2010: 150), sí sucede en el lexicón mental de los nativos.

\section{Conclusión}

Para concluir, se puede señalar que el estudio del léxico disponible de grupos de estudiantes de diferente nivel lingüístico en la LE nos permite sacar conclusiones sobre el desarrollo del tamaño de su léxico productivo en el área temática analizada. Así, contestamos a nuestra primera pregunta de investigación y podemos afirmar que la prueba de disponibilidad léxica puede servir como herramienta de evaluación de este aspecto del conocimiento léxico.

Sin embargo, la comparación del léxico disponible en esta área temática de los dos grupos de informantes con el PCIC ha revelado una de las limitaciones de la prueba de la disponibilidad léxica: la carencia, en las listas de respuestas, de clases de palabras diferentes de los sustantivos. Dicha carencia nos impide conocer con certeza cuáles son las palabras desconocidas y se debe principalmente a la naturaleza del centro de interés que se utiliza como estímulo. Por lo tanto, y para contestar a la segunda y a la tercera preguntas de investigación, pensamos que la prueba de disponibilidad léxica puede servir como prueba de diagnóstico tanto desde una perspectiva cuantitativa como cualitativa, a condición de que se haya seleccionado un estímulo que propicie la aparición de todas las clases de palabras o bien un estímulo que se extienda a subestímulos que estimen las asociaciones de palabras de diferentes categorías gramaticales.

No obstante, dicho análisis nos ha revelado información relacionada con la profundidad del conocimiento de las palabras ya adquiridas. Concretamente, se ha mostrado que algunas de las palabras adquiridas están almacenadas en el lexicón mental de los alumnos de una manera aislada, sin haberse formado redes semánticas. Estos datos son valiosos porque pueden orientar al enseñante mostrándole que debe enseñar el léxico de un modo que favorezca la creación de asociaciones semánticas de todos los tipos (paradigmáticas y sintagmáticas) entre las palabras ya adquiridas, así como entre las palabras adquiridas y las nuevas. 
En resumen, se ha comprobado que el léxico disponible refleja distintos aspectos del conocimiento léxico. De ahí que pueda servir como una herramienta de evaluación del tamaño del léxico productivo en el área temática analizada y también como prueba de diagnóstico del conocimiento productivo del léxico temático analizado a condición de que se haya seleccionado el estímulo adecuado.

\section{Bibliografía}

Bartol Hernández, J. A. (2006): «La disponibilidad léxica». RSEL, 36, 379-396. Caggiula, S. (2013): El español como lengua extranjera: un estudio de disponibilidad léxica y su aplicación a la enseñanza: https://books.google.gr/books?id $=$ i74XBAAAQBAJ\&pg=PA4\&lpg=PA4\&dq=disponibilidad+lexica+caggiu la (15-4-2019).

Carcedo González, A. (1998): «Sobre las pruebas de disponibilidad léxica para estudiantes de español LE». RILCE, 14(2), 205-224.

Carter, R. (1987): Vocabulary. Applied Linguistic Perspectives. Londres: Allen \& Unwin.

Cassany, D., Luna, M., Sanz, G. (1994): Enseñar lengua. Barcelona: Graó.

Consejo de Europa (2001): Marco común europeo de referencia para las lenguas: aprendizaje, enseñanza, evaluación. Madrid: MECD-Anaya.

Ferreira R., Echeverría, M. (2010): «Redes semánticas en el léxico disponible de inglés $L_{1}$ e inglés LE». Onomázein, 1, 21, 133-153.

Fries, C. (1945): Teacbing and learning English as a foreign language. Ann Arbor: University of Michigan Press.

Gozalo, P., Martín, M. (2008): Pruebas de nivel ELE. Modelos de examen para determinar el nivel de nuevos estudiantes. Madrid: SGEL.

Henriksen, B. (1999): «Three Dimensions of Vocabulary Development». Studies in Second Language Acquisition, 21, 303-317.

Hidalgo Gallardo, M. (2017): «La disponibilidad léxica como herramienta de evaluación interventiva del vocabulario en ELE: Aplicación en un contexto universitario sinohablante». SinoELE, 16, 43-63.

Higueras, M. (1996): «Aprender y Enseñar Léxico». En: Neus Sans, Lourdes Miquel (eds.), Cuadernos del Tiempo Libre. Colección Expolingua. Didáctica del español como lengua extranjera. Madrid: Fundación Actilibre, 3 , 111-126. 
Jiménez Catalán, R.M. (2002): «El Concepto de Competencia Léxica en los Estudios de Aprendizaje y Enseñanza de Segundas Lenguas». Atlantis, $24(2), 149-162$.

López González, A. M. (2010): «La Evaluación del Desarrollo de la Competencia Léxica en L2 por medio de la Disponibilidad Léxica». Redele, 18: https:// www.mecd.gob.es/dam/jcr:4f5777a6-d513-4715-910a 01c047353995/2010redele-18-o1lopez-pdf.pdf (15-4-2019).

López-Mezquita Molina, M. T. (2005): La evaluación de la competencia léxica. Tests de vocabulario: su fiabilidad y validez. Tesis doctoral inédita. Granada: Universidad de Granada.

López Morales, H. (1984): Enseñanza de la lengua materna. Lingüística para maestros de español. Madrid: Playor.

Meara, P. (1980): «Vocabulary acquisition: a Neglected Aspect of Language Learning». Language Teaching and Linguistics: Abstracts, 13, 221-246.

Meara, P. (1996): «The dimensions of lexical competence». En: Brown Gillian, Malmkjaer Kirsten, John Williams (eds.), Performance and Competence in Second Language Acquisition, Cambridge: CUP, 35-53.

Meara, P. (2007): «Simulating Word Associations in an L2; the Effects of Structural Complexity». Language Forum, 33(2), 13-31.

Nation, P. (1990): Teaching and learning vocabulary. Rowely: Newbury House.

Nation, P. (2001): Learning vocabulary in another language. Cambridge: CUP.

Palapanidi, K. (2016): «La disponibilidad léxica de aprendientes griegos de español como lengua extranjera en diferentes niveles lingüísticos». En: Pandís Pavlakis Efthimia, Haralambos Symeonidis, Slobodan Pajovic, Dimitrios Drosos, Victoria Kritikou (eds.) Estudios y bomenajes bispanoamericanos IV, España: Ediciones Clásicas, S.A., 305-313.

Palapanidi, K. (2019): «Manifestaciones de clusters y switches en el léxico disponible de aprendices griegos de ELE en diferentes niveles lingüísticos». RedELE, 28: https://marcoele.com/descargas/28/palapanidi-disponiblidadlexica griegos.pdf (15-4-2019).

Palapanidi K., Agustín Llach, M. P. (2017): «Análisis comparativo de la disponibilidad léxica de aprendientes griegos de español como lengua extranjera con la de estudiantes nativos». En: Pandís Pavlakis, Efthimia, Haralambos Symeonidis, Slobodan Pajovic, Dimitrios Drosos, Paul Chandler, Anthí Papageorgíou, Victoria Kritikou (eds.), Estudios y bomenajes bispanoamericanos V. Madrid: Ediciones Clásicas, 297-306. 
Paredes García, F. (2015): «Disponibilidad léxica y enseñanza de ELE: el léxico disponible como fuente curricular y como recurso en el aula». Linred, 13: http://www.linred.es/monograficos_pdf/LR_monografico13-articulo2.pdf (15 4-2019).

Read, J. (1997): «Vocabulary and Testing». En: Norbert Schmitt, Michael McCarthy (eds.), Vocabulary: Description, Acquisition and Pedagogy. Cambridge: CUP, 303-320.

Read, J. (2000): Assessing vocabulary. Cambridge: CUP.

Richards, J. (1976): «The Role of Vocabulary Teaching». TESOL Quarterly, 10(1), 77-89.

Ringbom, H. (1983): «Borrowing and Lexical Transfer». Applied Linguistics, 4, 207- 212.

Ringbom, H. (2007): Cross-linguistic Similarity in Foreign Language Learning. Clevedon/Buffalo/Toronto: Multilingual Matters Ltd.

Salazar García, V. (2004): «Acercamiento crítico a la selección objetiva de contenidos léxicos en la enseñanza de E/LE», Estudios de Lingüística, 18: 243-273.

Samper Padilla, J. A. (1998): «Criterios de edición del léxico disponible: sugerencias», Lingüística, 10, 311-333.

Sánchez Gómez, C. (2005): «Naturaleza gramatical del léxico disponible en informantes de español como lengua extranjera», Interlingüística, 16(2), 977986.

Sánchez-Saus Laserna, M. (2011): Bases semánticas para el estudio de los centros de interés del léxico disponible. Disponibilidad léxica de informantes extranjeros en las universidades andaluzas. Tesis dotoral inédita. Cádiz: Universidad de Cádiz.

Schmitt, N. (2000): Vocabulary in Language Teaching. Cambridge: CUP.

Šifrar Kalan, M. (2012): «Análisis comparativo de la disponibilidad léxica en español como lengua extranjera y lengua materna». Marcoele, 15: https:// marcoele.com/descargas/15/sifrar-disponibilidad_lexica.pdf (15-4 2019).

Šifrar Kalan, M. (2014): «Disponibilidad Léxica en Diferentes Niveles de Español/Lengua Extranjera». Studia Romanica Posnaniensia, 41(1), 63-84.

Tomé Cornejo, C. (2015): Léxico disponible. Procesamiento y aplicación a la enseñanza de ELE. Tesis doctoral inédita. Salamanca: Universidad de Salamanca. Wallace, M. (1982): Teacbing vocabulary. London: Heinmann Educational. 
Zuccalà, D. (2014): «Disponibilidad léxica del español como lengua extranjera: el caso brasileño». V Congreso Internacional de FIAPE, Cuenca, 25-28 de junio de 2014: http://www.educacionyfp.gob.es/dam/jcr:fe2606fb-edd5489f-8ac9 272b6bfo79e8/34disponibilidad\%2o-lexica-de-ele--zuccaladaniele-pdf.pdf" de-ele--zuccaladaniele-pdf.pdf (15-4-2019). 
Kiriakí Palapanidi

National and Kapodistrian University of Athens

\section{Lexical availability as an instrument for the lexical evaluation of Greek SFL learners}

Keywords: lexical availability, Greek learners of Spanish, lexical competence, evaluation of lexical competence

The purpose of this study is to show that the lexical availability test can be used as a tool for evaluation of foreign language learners' lexical competence. Evaluating the vocabulary knowledge of a learner is a complicated procedure, because knowing a word involves many different aspects. Despite the fact that the lexical availability test cannot evaluate all these, we believe that it can be a useful tool for evaluation of the size of productive thematic vocabulary with different purposes: evaluating progress in the knowledge of this aspect and identifying gaps in the learners' vocabulary knowledge. In order to test this, we analyse the responses in a lexical availability task of two groups of Greek SFL learners of two different linguistic levels, $\mathrm{B}_{1}$ and $\mathrm{C}_{1}$ and compare their responses to the prompt $L a$ casa. Then, we compare their responses with the Curricular Plan of the Instituto Cervantes. The results show us that the lexical availability test can be a useful tool for evaluation of the size of productive thematic vocabulary, and it could reveal gaps in the learners' knowledge in the analysed area of interest on condition that a centre of interest which permits the production of all types of words has been selected. 
Kiriakí Palapanidi

Narodna in Kapodistrijska univerza $v$ Atenah

\section{Leksikalna razpoložljivost kot sredstvo za leksikalno vrednotenje grških učencev španščine kot tujega jezika}

Ključne besede: leksikalna razpoložljivost, grški učenci španščine kot tujega jezika, leksikalna zmožnost, vrednotenje leksikalne zmožnosti

V prispevku nameravamo pokazati, da test leksikalne razpoložljivosti lahko služi kot sredstvo za leksikalno vrednotenje učencev tujih jezikov. Ker gre pri leksikalni zmožnosti za večplasten pojav, je razumljivo, da je njeno vrednotenje zelo zahtevno. Čeprav test leksikalne razpoložljivosti ne more oceniti vseh vidikov leksikalne zmožnosti, menimo, da bi lahko bil uporabno sredstvo za vrednotenje obsega produktivnega tematskega besedišča. Služi namreč dvema različnima namenoma: po eni strani ovrednoti razvoj danega vidika znanja besedišča pri učencih tujih jezikov, po drugi pa razkrije morebitne vrzeli $\mathrm{v}$ besedišču na analiziranem tematskem polju. Da bi to potrdili, smo v dveh skupinah grških učencev španščine kot tujega jezika, eni na ravni $\mathrm{B} 1$, drugi pa $\mathrm{C}_{1}$, izvedli test leksikalne razpoložljivosti, nato pa primerjali njune odgovore na semantičnem polju »Hiša«. Nato smo odgovore obeh skupin primerjali še z desetim poglavjem (»Bivališče«) »Specifičnih znanj« iz Učnega načrta Instituta Cervantes (Plan Curricular del Instituto Cervantes, PCIC). Rezultati kažejo, da lahko test leksikalne razpoložljivosti služi kot sredstvo za vrednotenje razvoja obsega produktivnega besedišča $\mathrm{z}$ analiziranega tematskega polja in da bi lahko razkril tudi vrzeli v znanju besedišča na danem tematskem polju, vendar zgolj pod pogojem, da se uporabi na semantičnem polju, ki omogoča produkcijo vseh besednih vrst. 